\title{
Costes intangibles de la dependencia alcohólica desde la perspectiva de los pacientes y sus familiares: un estudio de valoración contingente
}

\section{Intangible costs of alcohol dependence from the perspective of patients and their relatives: A contingent valuation study}

\author{
Jacinto Mosquera Nogueira*, Eva Rodríguez-Míguez**. \\ * Physician. Alcohol Unit of Vigo (Galician Health Service). Spain. ** Economist. Departament of Applied Economics. \\ University of Vigo. Spain
}

\section{Resumen}

La dependencia alcohólica produce múltiples problemas no sólo a la persona que la padece sino también a su entorno. En este estudio se utiliza la valoración contingente para valorar los efectos intangibles de la dependencia alcohólica, desde la perspectiva de las personas directamente implicadas: pacientes y familiares. Se ha entrevistado a 145 pacientes y 61 familiares. Los efectos intangibles de la dependencia alcohólica se obtienen a partir de la disponibilidad a pagar por un hipotético tratamiento para la dependencia, ante dos escenarios de éxito $(50 \%$ y $100 \%)$. La disponibilidad a pagar media mensual de la población alcohólica es de $129 €$ y $168 €$, respectivamente, por los tratamientos con un $50 \%$ y un $100 \%$ de éxito. La disponibilidad de los familiares es mayor en ambos escenarios (307€ y 420€, respectivamente), lo cual podría ser explicado por su mayor percepción de los problemas familiares, laborales y de salud generados por la dependencia alcohólica. El análisis de regresión realizado muestra que la eficacia del tratamiento, la renta personal y tener un deterioro moderado de la salud influyen positivamente en la disponibilidad a pagar de los pacientes, e influye negativamente estar desanimado y deprimido. Los resultados de este estudio pueden ser aplicados a estudios de evaluación económica cuyo objetivo es medir los beneficios de programas destinados a reducir la prevalencia de la dependencia alcohólica. Los costes intangibles estimados pueden ser añadidos a los costes directos e indirectos habitualmente utilizados.

Palabras clave:Alcohol; Dependencia; Familia; Valoración contingente; Disposición a pagar.

\begin{abstract}
Alcohol dependence causes multiple problems not only for the person suffering dependence but also for others. In this study, the contingent valuation method is proposed to measure the intangible effects of alcohol dependence from the perspective of the persons directly involved: the patients and their relatives. Interviews were conducted with 145 patients and 61 relatives. Intangible effects of alcohol dependence were determined based on willingness to pay for a hypothetical treatment for dependence, with different success scenarios $(50 \%$ and $100 \%)$. The mean monthly willingness to pay among the alcohol-dependent population was $€ 129$ and $€ 168$, respectively, for the treatments with $50 \%$ and $100 \%$ success. The willingness to pay of relatives was greater in both scenarios (€307 and $€ 420$, respectively), which could be explained by their greater perception of the family, labour, and health problems resulting from alcohol dependence. Regression analysis showed that patients' willingness to pay is positively related to treatment efficacy, personal income and moderate health deterioration, and negatively related to feeling discouraged and depressed. The results from this study can be applied to economic valuation studies that aim to measure the benefits of programs intended to reduce the prevalence of alcohol dependence. The intangible costs estimated can be added to the direct and indirect costs commonly used.

Keywords: Alcohol; Dependence; Family; Contingent valuation; Willingness to pay.
\end{abstract}


$\mathrm{E}$ 1 consumo excesivo de bebidas alcohólicas es muy prevalente. Se estima que cerca del $15 \%$ del la población europea consume alcohol excesivamente (Rehm et al., 2004) y entre el 1.2 - 3\% padece dependencia alcohólica (Anderson y Baumberg, 2006; Rehm, Rehm, Shield, Gmel y Gual, 2013). Los efectos del consumo excesivo de alcohol tiene innumerables costes económicos directos e indirectos (Anderson et al., 2006; Baumberg, 2010). Los costes directos hacen referencia a gastos que se hubieran podido emplear para cosas más productivas, principalmente los costes derivados de mayores gastos médicos (Johansson et al., 2006). Los costes indirectos se refieren, principalmente, a la pérdida de recursos causada por la menor participación en el mercado de trabajo y la menor productividad de los trabajadores con problemas de alcohol (Petersen et al, 2005).

La dependencia alcohólica también tiene numerosos costes intangibles, o no-financieros, tales como una menor esperanza de vida y una calidad de vida reducida (dolor, sufrimiento, problemas de salud física, etc.), tanto para la persona dependiente como para las personas de su entorno. "Estos costes no son financieros porque no tienen un valor monetario, en el sentido de que no se puede vender o intercambiar el dolor. No obstante, los individuos y la sociedad estarían dispuestos a pagar algo para evitarlos, lo que significa que tienen un valor no-financiero" (Baumberg, 2010). La mayoría de los estudios que han analizado los costes intangibles se centran en los efectos sobre la salud del bebedor en términos de mortalidad (Collins y Lapsley, 2008; John et al., 2013) y de calidad de vida. Una de las medidas de calidad de vida usadas con mayor frecuencia es la de años de vida ajustados por calidad. Esta medida se ha aplicado para evaluar tanto el impacto de la dependencia alcohólica (Kraemer et al., 2005; Maheswaran, Petrou, Rees y Stranges, 2013; Petrie, Doran, Shakeshaft y Sanson-Fisher, 2008; Saarni et al., 2007; Sanderson, Andrews, Corry y Lapsley, 2004; Stouthard, Essink-Bot y Bonsel, 2000) como el beneficio de las intervenciones encaminadas a su tratamiento o prevención (Chisholm, Rehm, Van Ommerem y Monteiro, 2004; Corry, Sanderson, Issakidis, Andrews y Lapsley, 2004; Mortimer y Segal, 2005; Parrott, Godfrey, Heather, Clark y Ryan, 2006; UKATT Research Team, 2005). En España, si bien los manuales clínicos proporcionan una amplia descripción de las consecuencias intangibles, pocos estudios se han centrado en la medición de estos efectos. La reciente revisión de García-Pérez et al., (2014) que encontró dos estudios que cuantifican el impacto del alcoholismo en la calidad de vida (Fernández et al., 2010; Grandes, Montoya, Arietaleanizbeaskoa, Arce y Sanchez, 2011) y el estudio de Mosquera y Rodríguez-Míguez (2015) proporcionan nueva evidencia empírica de los efectos de la dependencia alcohólica en la calidad de vida del dependiente y de las personas de su entorno.

Sin embargo, los efectos intangibles sobre el bienestar causados por el alcohol van más allá de los efectos direc- tos sobre la salud del bebedor. Así, la dependencia alcohólica tiene efectos adicionales sobre el bebedor, tales como el sufrimiento, el aislamiento, los problemas familiares, la exclusión social, etc. Además, esta enfermedad tiene efectos considerables sobre el entorno del bebedor (Laslett et al., 2010). Si bien el alcohol se considera la sustancia adictiva que inflige el mayor daño a los demás (Nutt, King y Phillips, 2010), pocos estudios han analizado estos efectos intangibles. Salvo algunos estudios que han estimado la calidad de vida perdida por los familiares (Jarl et al., 2008; Mosquera et al., 2015), la mayoría de las investigaciones en este campo se centran en la medición de los costes directos e indirectos. Así, las investigaciones se han concentrado en el estudio del síndrome de alcoholismo fetal y el impacto del abuso de alcohol en las víctimas de delitos y de accidentes de tráfico, utilizando el coste de la enfermedad como el método primario de medición (para una revisión de estas estudios, ver Navarro, Doran y Shakeshaft, 2011). La no consideración de los efectos intangibles del consumo de alcohol puede resultar en una subestimación significativa de los efectos de la enfermedad, así como de los beneficios asociados al tratamiento.

Los estudios de valoración contingente que utilizan el método de la disponibilidad a pagar (DAP) han demostrado que es una herramienta útil para la evaluación de los efectos de determinados tratamientos que proporcionan beneficios más allá de la salud. El método DAP permite la valoración de los costes intangibles de la dependencia alcohólica, basada en la cantidad máxima que una persona está dispuesta a pagar para reducir, eliminar o evitar la situación. Esta metodología ha sido ampliamente aplicada en la valoración de las consecuencias para la salud (Byrne, O'Malley y Suarez-Almazor, 2005; Fautrel et al., 2007; Greenberg, Bakhai, Neumann y Cohen, 2004; Gueylard-Chenevier y Leloier, 2005; Pinto-Prades, Farreras y de Bobadilla, 2008), así como en procedimientos clínicos (Bergmo y Wangberg, 2007; Boonen et al., 2005; He et al., 2007; Jimoh, Sofola, Petu y Okorosobo, 2007; Sadri, Mackeigan, Leite y Einarson, 2005; Walsh y Bartfield, 2006; Whynes, Frew y Wolstenholme, 2003; Yasunaga, Ide, Imamura y Ohe, 2006; Unutzer et al., 2003) (para una revisión de estudios antes de 2002, ver Smith, 2003). La aplicación de esta metodología al área de las drogas en general (Bishai et al., 2008; Tang, Liu, Chang y Chang, 2007; Zarkin, Cates y Bala, 2000) y a la dependencia alcohólica en particular ha sido muy limitada. Hasta donde sabemos, sólo dos estudios de valoración contingente publicados han utilizado el método DAP para medir los efectos del consumo abusivo de alcohol. Jeanrenaud and Pellegrini (2008) utilizaron una muestra 236 sujetos de la población general suiza para averiguar la DAP por un tratamiento curativo de la dependencia alcohólica de un hipotético familiar coviviente. Petrie, Doran y Shakeshaft (2011) utilizaron una muestra de la población general australiana para averiguar la DAP para reducciones del $10 \%$ y del $20 \%$ en los 
daños causados por el alcohol en la población. Sin embargo, no conocemos ningún estudio que hubiera obtenido la DAP de los propios pacientes o de sus familiares. Puede ser importante, porque hay abundante evidencia empírica que muestra que las preferencias de la población general y de las personas directamente implicadas pueden ser muy diferentes (Brazier et al., 2005; Gabriel et al., 1999; Mann, Brazier y Tsuchiya, 2009; Ubel, Loewenstein y Jepson, 2003).

El objetivo de este estudio es cuantificar los costes intangibles de la dependencia alcohólica, desde la perspectiva de los propios pacientes y de sus familiares en España en 2010. En la línea de los estudios mencionados anteriormente, nuestro estudio aplica el método DAP para estimar estos efectos intangibles en un sentido amplio, no sólo los efectos en la salud. Sin embargo, a diferencia de ellos nuestro estudio mide esos efectos desde la perspectiva de las personas directamente implicadas, quienes fueron personalmente entrevistadas por el primer autor.

\section{Materiales y métodos}

\section{Muestras}

Contactamos con los pacientes y sus familiares en una unidad de tratamiento de alcohol dentro del Servicio Nacional de Salud. Esta unidad trata a pacientes con dependencia alcohólica del área sanitaria de Vigo (España). La muestra de pacientes, quienes cumplían los criterios del DSM-4R para la dependencia alcohólica, incluía a todos los que vinieron a consulta durante dos meses, a partir de enero de 2010. La participación en el estudio era voluntaria y anónima. Los criterios de exclusión eran: negarse a participar en la primera consulta en el centro, intoxicación alcohólica aguda o trastorno mental sin tratar en el momento de la entrevista, y deterioro cognitivo que obstaculizaba la comprensión del cuestionario (en opinión del terapeuta). La muestra de los familiares incluyó a todas las personas que acompañaron a los pacientes a la entrevista el día que se llevó a cabo. Si los pacientes entraron a consulta solos en el momento de inclusión en el estudio, la persona que normalmente les acompañaba (si la hubo) fue contactada por teléfono para invitarle a participar voluntariamente. No había criterio de exclusión salvo el rechazo a participar. Durante el periodo de reclutamiento, 161 pacientes acudieron a consulta. Se excluyeron a dos pacientes por intoxicación alcohólica, dos por enfermedad mental no tratada y seis por deterioro cognitivo. Además, cinco pacientes fueron excluidos del los análisis porque no proporcionaron una DAP. Un sujeto se negó a participar. Sólo en 66 casos, pudimos entrevistar a un familiar (en los restantes casos, no había ningún pariente implicado en el proceso de tratamiento). Un familiar se negó a participar y cuatro fueron excluidos porque no proporcionaron una DAP. El primer co-autor entrevistó personal e independientemente a los participantes, para abordar los potenciales problemas que pudiesen surgir durante la entrevista.

\section{Cuestionario}

En la primera parte de la entrevista, los participantes fueron informados acerca de la naturaleza voluntaria y anónima de la misma y se obtuvo su consentimiento informado para participar. Al mismo tiempo, se explicó a los participantes que los escenarios propuestos eran hipotéticos y que sus respuestas de ninguna manera influirían en la atención recibida. En la segunda parte de la entrevista, se describió el siguiente escenario a los sujetos:

"Imagine una situación hipotética, una situación que no es real. Supongamos que hay un nuevo tratamiento para resolver los problemas relacionadas con el alcohol. Este tratamiento no es siempre eficaz. En 5 de cada 10 personas (es decir, la mitad de los pacientes tratados) es eficaz, es decir, dejan de beber alcohol y no desean hacerlo. En la otra mitad de los pacientes, el tratamiento no es eficaz. Los efectos del tratamiento permanecen durante un año. Después de un año, el sujeto tendría que recibir el tratamiento de nuevo con la misma probabilidad de éxito. El tratamiento no es gratis, es decir, no está financiado por el Servicio Nacional de Salud. ¿Cuál es el importe anual máximo que pagarías para recibir tal tratamiento? Piensa tu respuesta con calma. Debes tener en cuenta tu nivel de ingresos. Por favor, ten en cuenta que este pago implicaría renunciar al consumo de otros bienes o reduciría tu capacidad para ahorrar dinero".

Además, como proponen Blumenschein, Johannesson, Yokoyama y Freeman (2001), se incluyó una pregunta confirmatoria. Después de que los participantes habían ofrecido las cantidades máximas que estarían dispuestos a pagar por el tratamiento, se les pidió elegir entre dos respuestas: "Estoy absolutamente seguro de que lo pagaría” " "creo que yo pagaría pero no estoy seguro". Si optaron por la segunda respuesta, se les pidió que proporcionaran de nuevo la cantidad que estaban seguros de pagar. A continuación, se propuso otro escenario en el que la eficacia del hipotético tratamiento fue del $100 \%$, pero el paciente tendría que seguir el tratamiento indefinidamente, porque de lo contrario sufriría una recaída, volviendo a la situación inicial. Al igual que en el escenario anterior, se preguntó a los participantes cuál era lo máximo que estarían dispuestos a pagar mensualmente, seguido de la pregunta confirmatoria.

En la tercera parte de la entrevista, se les pidió a los participantes una opinión subjetiva sobre las consecuencias de su dependencia alcohólica en cuatro áreas: salud, relaciones familiares, consecuencias laborales, y problemas legales. Las posibles respuestas en cada caso eran fueron casi ninguna, moderadas/algunas y severas/muchas. También conocemos la fecha de inicio del tratamiento real así como los niveles de consumo (medidos en unidades de bebida estándar), en un día normal, en esa fecha. Seguidamente, se hicieron las preguntas típicas sociodemográficas a los participantes. Finalmente, se pidió a los pacientes así como a los familiares que 
completaran el Short-Form Health Survey (SF-36; Forma Abreviada de Encuesta de Salud), un cuestionario genérico de la calidad de vida relacionada con la salud (información necesaria para otro estudio en curso).

\section{Análisis estadístico}

El coste intangible de la dependencia alcohólica se estimó sobre la base de los valores de la media y la mediana proporcionados por los participantes después de la pregunta confirmatoria para los dos escenarios de éxito. A continuación, se realizó una regresión lineal para identificar las variables correlacionadas con la DAP. La variable independiente eran las DAP proporcionadas por los participantes después de la pregunta confirmatoria y las variables explicativas eran las variables que, a priori, podrían estar relacionadas con las DAP. Se utilizó un modelo de regresión con efectos aleatorios para tener en cuenta que los participantes proporcionaban dos respuestas, una para el tratamiento con un $50 \%$ de probabilidad de éxito y la otra para el tratamiento con un $100 \%$ de probabilidad de éxito.

\section{Análisis de validez}

Existe un consenso de que los estudios de valoración contingente, como mínimo, deben mostrar una correlación positiva entre la DAP y el nivel de ingresos. Por ello, el signo del coeficiente de regresión para esta variable se utiliza como la prueba de validez teórica. La falta de literatura previa acerca de la DAP en una población de dependientes del alcohol representa un reto considerable a la formulación de las hipótesis respecto de las restantes variables. En cualquier caso, parece razonable que, ceteris paribus, cuanto peor sean las consecuencias de la dependencia, mayor debería ser la DAP por el tratamiento. Otro resultado esperado es que la DAP sea sensible a la cantidad y/o calidad del bien (Arrow et al., 1993), conocido como sensibilidad a la escala. En nuestro estudio, analizamos si la DAP para el tratamiento con $100 \%$ de éxito era o no significativamente mayor que la DAP por el tratamiento con $50 \%$ de éxito. La ausencia de apoyo para esta hipótesis plantearía serias dudas sobre la validez de los resultados (Diamond y Haussman, 1994).

La conformidad con los dos análisis precedentes de validez es una condición necesaria pero no suficiente para garantizar la validez de los resultados. La validez de criterio es la más importante prueba de validez, porque analiza hasta qué punto los resultados para un escenario hipotético se ajustan a los obtenidos en una transacción real. Ya que se propuso un tratamiento hipotético en nuestro estudio como un mecanismo para la obtención de los costes intangibles de la dependencia alcohólica, la validez de criterio no se puede poner a prueba. La imposibilidad de poner a prueba la validez de criterio es común a otros estudios de DAP (de hecho, la falta de un verdadero mercado es una del razones que justifica la realización del estudio de la DAP). Sin embargo, esta prueba es relevante porque las diferencias entre la DAP en una situación real y una hipotética pueden ser muy grandes. El estudio de Blumenschein et al. (2001) sobre la DAP para un tratamiento de asma encontró que la sobreestimación obtenida del escenario hipotético (en comparación con un escenario de compra real) se corrigió preguntando a los entrevistados si estaban absolutamente seguros de que harían el pago que habían mencionado. Por esta razón, para minimizar la diferencia potencial entre la DAP real y la hipotética, hicimos la pregunta confirmatoria que evalúa la certeza con la que los entrevistados aseguran que pagarían las cantidades que inicialmente proporcionaron.

\section{Resultados}

\section{Descripción de las muestras}

La tabla 1 resume las características de los 145 pacientes y 61 familiares seleccionados. Los varones predominan en la muestra de pacientes y cerca de la mitad de ellos convive con su cónyuge o una pareja. La media de los ingresos personales es de $766 €$, y 20 sujetos dijeron que sus ingresos eran de $0 €$ (en siete casos, los ingresos familiares también eran de $0 €)$. Comparamos la información de la muestra de pacientes para sexo, edad media y educación con la información proporcionada por la institución para todos los pacientes bajo su cuidado, no encontrando diferencias significativas. Más de la mitad de la muestra de parientes la formaban mujeres, cónyuges de la persona dependiente. La tabla 1 muestra el estado emocional (abatido y deprimido) de la persona dependiente durante las últimas cuatro semanas (obtenido del SF-36) y el porcentaje de ellos que tenía apoyo familiar (se considera que el paciente tiene el apoyo de su familia si fue posible contactar con un miembro de la familia para su inclusión en el estudio).

Respecto a la percepción de los entrevistados sobre las consecuencias de la dependencia alcohólica, parece que tanto los pacientes como los familiares coincidieron en que los problemas familiares, seguido de los problemas de salud, eran los más frecuentes. Sin embargo, con la excepción de los problemas legales, los familiares percibían significativamente más problemas que los expresados por los pacientes (esta conclusión se mantiene cuando comparamos la muestra de familiares con la submuestra de 61 pacientes cuyo pariente fue entrevistado).

Todos los datos están disponibles mediante petición al autor de correspondencia.

\section{Resultados DAP}

La tabla 2 muestra la media y la mediana de la DAP y la figura 1 proporciona la distribución de la DAP. La DAP media mensual para un tratamiento con $50 \%$ de eficacia fue de $135 €$ después de la primera pregunta y de $129 €$ después de la pregunta confirmatoria. En 23 casos, la respuesta fue de $0 €$. La DAP mensual por el tratamiento con $100 \%$ de efec- 
Tabla 1. Descripción de muestras de pacientes y familiares

\begin{tabular}{|c|c|c|c|}
\hline & & $\begin{array}{c}\text { Pacientes } \\
(\mathrm{n}=145)\end{array}$ & $\begin{array}{c}\text { Familiares } \\
(n=61)\end{array}$ \\
\hline Sexo (\% varones) & & 69.66 & 18.03 \\
\hline \multirow{4}{*}{ Distribución por edad (\%) } & 18 a 29 años & 5.59 & 6.56 \\
\hline & 30 a 44 años & 30.34 & 31.15 \\
\hline & 45 a 59 años & 48.28 & 39.34 \\
\hline & 60 años o mayor & 15.86 & 22.95 \\
\hline Ingresos personales promedios ( $€ /$ mes) & & 765.93 & 854.16 \\
\hline Ingresos familiares promedios (€/mes) & & 1301.03 & 1826.57 \\
\hline \multirow{3}{*}{ Nivel de educación (\%) } & Primaria o menos & 66.9 & 68.85 \\
\hline & Secundaria & 25.52 & 16.39 \\
\hline & Educación superior & 7.59 & 14.75 \\
\hline Vive con pareja (\%) & & 45.52 & 85.24 \\
\hline \multirow{2}{*}{ Abatido y deprimido (\%) } & Nada/una parte del tiempo & 37.93 & 50.82 \\
\hline & Algo/la mayor parte/todo el tiempo & 62.07 & 49.18 \\
\hline \multirow{3}{*}{ Consecuencias familiares (\%) } & Casi ninguna & 17.93 & 8.2 \\
\hline & Moderada/algunos problemas & 36.55 & 31.15 \\
\hline & Grave/muchos problemas & 45.52 & 60.66 \\
\hline \multirow{3}{*}{ Consecuencias en la salud (\%) } & Casi ninguna & 31.03 & 19.67 \\
\hline & Moderada/algunos problemas & 40.69 & 44.26 \\
\hline & Grave/muchos problemas & 28.28 & 36.07 \\
\hline \multirow{3}{*}{ Consecuencias legales (\%) } & Casi ninguna & 69.66 & 78.69 \\
\hline & Moderada/algunos problemas & 15.86 & 8.2 \\
\hline & Grave/muchos problemas & 14.48 & 13.11 \\
\hline \multirow{3}{*}{ Consecuencias laborales (\%) } & Casi ninguna & 69.66 & 52.46 \\
\hline & Moderada/algunos problemas & 17.24 & 22.95 \\
\hline & Grave/muchos problemas & 13.10 & 24.59 \\
\hline \multirow{3}{*}{ Ingesta de alcohol antes del tratamiento (\%) } & «4 unidades/día (hombres)/ «3 (mujeres) & 12.41 & \\
\hline & $>4$ y<8 (hombres) / >3 y <6 (mujeres) & 18.62 & \\
\hline & >8 unidades/día (hombres)/ >6 (mujeres) & 68.97 & \\
\hline \multirow{5}{*}{ Duración del tratamiento (meses) } & $<4$ & 12.41 & \\
\hline & $4-6$ & 7.59 & \\
\hline & $7-12$ & 15.17 & \\
\hline & $12-24$ & 35.86 & \\
\hline & $>24$ & 28.97 & \\
\hline \multirow[t]{3}{*}{ Tiene apoyo familiar (\%) } & & 42.76 & \\
\hline & Cónyuge & & 67.7 \\
\hline & $\mathrm{Hijo/a}$ & & 4.6 \\
\hline \multirow[t]{3}{*}{ La relación con el dependiente (\%) } & Hermano/a & & 10.8 \\
\hline & Progenitores & & 12.3 \\
\hline & Otro & & 4.6 \\
\hline
\end{tabular}

tividad fue de $168 €$, rechazando la existencia de la insensibilidad a la escala. Puesto que sólo un individuo cambió su respuesta después de la pregunta confirmatoria, la DAP final era prácticamente igual que antes. En 22 casos, la respuesta fue de $0 €$.

Entre los pacientes que no estaban dispuestos a pagar nada, no hay ninguna evidencia de que sus respuestas puedan ser consideradas respuestas de "protesta". Para empezar, el 55\% de los entrevistados con una DAP de cero por el tratamiento con $100 \%$ de éxito no tenía ingresos personales y el $29 \%$ tampoco tenía ingresos familiares (se las arreglaban con la ayuda de otras personas o de instituciones no gubernamentales). Estos porcentajes se reducen ligeramente (a $52 \%$ y $27 \%$, respectivamente) cuando el tratamiento tenía una tasa de éxito del $50 \%$. Además, si examinamos sólo los participantes que tenían ingresos personales, los ingresos medios son un $23 \%$ mayores entre los tenían una DAP positiva, en comparación con aquellos que dieron una respuesta de DAP de cero. Finalmente, los participantes que dieron una DAP de cero mencionan su bajo nivel de ingresos como la razón de esta respuesta. Por ello, creemos que no existe una clara justificación para considerar estas respuestas como respuestas "protesta" y se han incluido en los análisis. 
Tabla 2. Valores de la media y la mediana de la disponibilidad para pagar (DAP) mensual para pacientes y familiares

\begin{tabular}{lcccccc}
\hline & \multicolumn{3}{c}{ Pacientes $(\mathrm{n}=\mathbf{1 4 5})$} & & \multicolumn{2}{c}{ Familiares $(\mathrm{n}=\mathbf{6 1})$} \\
\hline & Media (error estándar) & Mediana (min, max) & $\begin{array}{c}\text { Percentiles } \\
\mathbf{2 5} \text { y 75 }\end{array}$ & Media (error estándar) & Mediana (min, max) & $\begin{array}{c}\text { Percentiles } \\
\mathbf{2 5} \text { y 75 }\end{array}$ \\
\hline DAP inicial 50\% éxito & $135.41(14.06)$ & $100(0-1000)$ & $30-200$ & $322.95(48.70)$ & $200(0-2000)$ & $80-400$ \\
DAP final 50\% éxito & $128.95(14.01)$ & $90(0-1000)$ & $30-150$ & $306.72(48.87)$ & $200(0-2000)$ & $55-300$ \\
DAP inicial 10\% éxito & $167.59(18.05)$ & $100(0-100)$ & $30-200$ & $420.25(65.21)$ & $300(0-2000)$ & $100-475$ \\
DAP final 100\% éxito & $167.53(18.05)$ & $100(0-1000)$ & $30-200$ & $420.25(65.21)$ & $300(0-2000)$ & $100-475$ \\
\hline
\end{tabular}

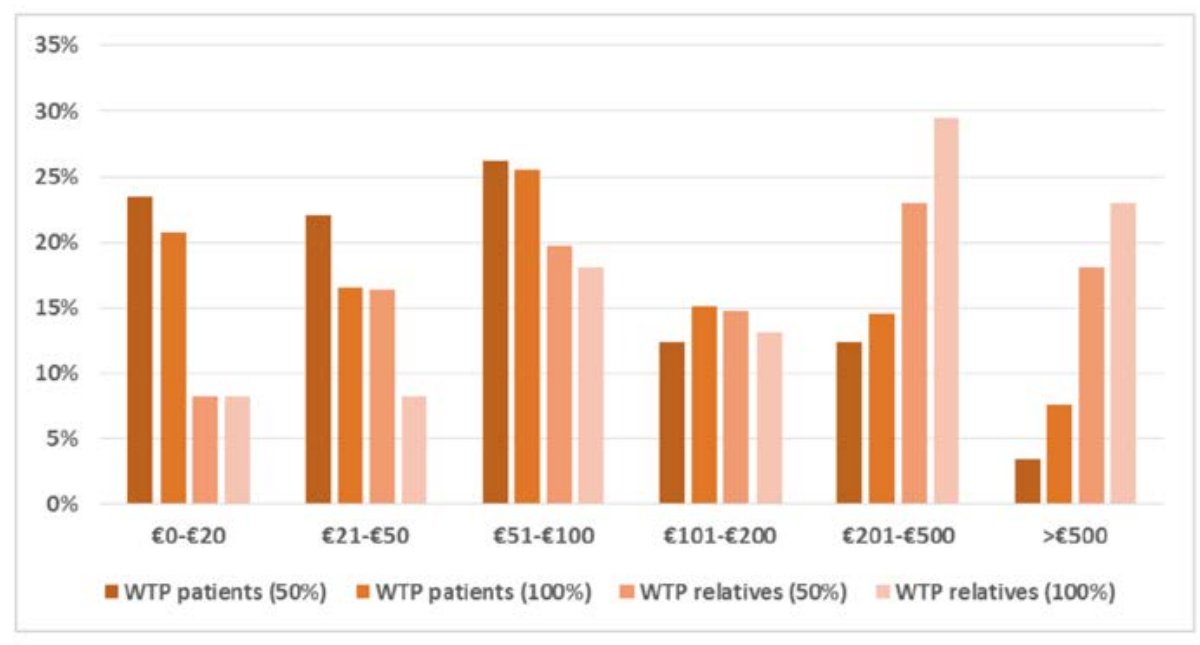

Figura 1. Distribución de la disponibilidad para pagar (DAP)

La DAP para la muestra de los familiares era significativamente mayor, con una DAP media mensual de $307 €$ cuando la eficacia del tratamiento era del $50 \%$ y una DAP media mensual de $420 €$ cuando la eficacia era del $100 \%$. Sólo cuatro familiares dieron una DAP de cero. La mediana es inferior a la media pero muestra el mismo patrón, con valores mayores para el tratamiento del $100 \%$ de éxito que para el tratamiento del $50 \%$ de éxito y valoraciones superiores por parte de los familiares que de los pacientes.

\section{Determinantes de la DAP}

La tabla 3 muestra los resultados del análisis de regresión realizado para identificar los posibles determinantes de la DAP de los pacientes. La DAP se correlaciona positivamente con la eficacia del tratamiento (sensibilidad a la escala). En este sentido, los entrevistados estaban dispuestos a pagar $39 €$ adicionales para un tratamiento que garantizara el éxito, en comparación con uno con sólo un $50 \%$ de tasa de éxito. Los ingresos personales también correlacionaban positivamente con la DAP, apoyando la validez teórica de los resultados. La DAP también estaba positivamente relacionada con el apoyo familiar (se pudo contactar con un familiar implicado en el tratamiento) y negativamente con sentirse abatido y deprimido durante las últimas cuatro semanas.
Respecto a los efectos del alcoholismo en la salud, se ha encontrado que las personas para quienes la dependencia alcohólica ha causado problemas moderados de salud estaban dispuestas a pagar $108 €$ más que las personas que apenas tenían problemas de salud. Sin embargo, cuando la dependencia alcohólica había causado graves problemas de salud, la DAP, si bien positiva, no era significativa. En todo caso, el resultado que a priori podría parecer el más sorprendente es la correlación negativa entre la presencia de graves problemas familiares y la DAP. Este resultado, combinado con el hecho de que la DAP se relaciona negativamente con el sentimiento de abatimiento y depresión, puede estar relacionado con la influencia de las expectativas de autoeficacia del sujeto. En otras palabras, para pagar más por un tratamiento, debe haber algún grado de optimismo acerca de la posibilidad de éxito, que podría ser menos plausible en situaciones familiares muy deterioradas. Sin embargo, estos resultados se mantienen incluso para el tratamiento con el $100 \%$ de probabilidad de éxito, lo que puede indicar una motivación limitada para mejorar la vida entre los pacientes en situaciones muy deterioradas.

La variable "ingesta de alcohol" (véase la tabla 1) ha sido excluida del análisis de regresión porque se refiere a la fecha de inicio del tratamiento real, lo cual no es representativo de la situación actual. En cualquier caso, se estimó la 
Tabla 3. Determinantes de la disposición mensual a pagar (DAP)

\begin{tabular}{|c|c|c|c|}
\hline & Coeficiente & Valor $\mathbf{P}$ & $95 \%$ IC \\
\hline Eficacia del tratamiento (Ref. 50\%) & 38.59 & .001 & $22.67-54.50$ \\
\hline Sexo (Ref. hombre) & 1.90 & .955 & $-63.86-67.65$ \\
\hline Edad & -1.23 & .430 & $-4.29-1.83$ \\
\hline \multicolumn{4}{|l|}{ Educación (Ref. primaria o menos) } \\
\hline Secundaria & -2.55 & .944 & $-73.26-68.16$ \\
\hline Ingresos personales mensuales & 0.10 & .001 & $0.04-0.15$ \\
\hline \multicolumn{4}{|l|}{ Consecuencias para la salud (ref. casi ninguna) } \\
\hline Moderadas/algunas & 108.29 & .002 & $40.71-175.87$ \\
\hline Graves/muchas & 23.98 & .562 & $-57.08-105.03$ \\
\hline \multicolumn{4}{|l|}{ Consecuencias para la familia (ref. casi ninguna) } \\
\hline Graves/muchas & -70.97 & .099 & $-155.42-13.47$ \\
\hline \multicolumn{4}{|l|}{ Consecuencias legales (ref. casi ninguna) } \\
\hline Moderadas/algunas & -8.42 & .855 & $-98.78-81.94$ \\
\hline Graves/muchas & 25.05 & .591 & $-66.32-116.42$ \\
\hline \multicolumn{4}{|l|}{ Consecuencias Laborales (Ref. casi ninguna) } \\
\hline Moderadas/algunas & -24.80 & .580 & $-112.77-63.14$ \\
\hline Graves/muchas & -7.02 & .888 & $-104.96-90.92$ \\
\hline Abatido y deprimido (ref. ninguno /-Un poco) & -74.56 & .015 & $-134.63--14.50$ \\
\hline Duración del tratamiento & -0.46 & .769 & $-3.54-2.62$ \\
\hline Tiene apoyo de la familia (Ref. sin apoyo) & 54.34 & .077 & $-5.90-114.58$ \\
\hline Constante & 151.06 & .173 & $-66.08-368.20$ \\
\hline
\end{tabular}

Nota. $R_{2}=$ 0,265. Número de participantes, 145; número observaciones, 290

regresión con esta variable y no era significativa, obteniendo resultados similares en el resto de las variables. Se estimó también el modelo excluyendo a los pacientes que no tenían ingresos personales. Se obtuvieron resultados similares respecto al signo y la significación de los parámetros, salvo que las consecuencias familiares severas no eran significativas $(p=.127)$.

Los resultados de la regresión realizada sobre los datos de los familiares (no se muestran) indican que ninguna de las variables examinadas influye significativamente en la DAP, a excepción de la probabilidad de éxito y los ingresos (ambos significativos en el nivel del $5 \%$ de éxito). En cualquier caso, debemos ser muy cautelosos con estos resultados, dado el tamaño pequeño de la muestra de los parientes.

\section{Discusión}

El objetivo de este estudio era obtener una valoración monetaria de los costes intangibles de la dependencia alcohólica a través de un estudio de valoración contingente llevado a cabo con los pacientes y sus familiares. Si bien, hasta donde nosotros sabemos, no se había realizado ningún estudio previo de estas características, el estudio con la mayor similitud al nuestro es el de Jeanrenaud et al. (2007), llevado a cabo con una muestra de la población general suiza. Los autores encontraron que la DAP media por un tratamiento curativo para la dependencia alcohólica de un hipotético familiar conviviente representaba aproximadamente el $7 \%$ del promedio mensual de los ingresos de la unidad familiar, un porcentaje que es significativamente inferior al obtenido en nuestra muestra de familiares (23\% de los ingresos). Esta diferencia puede reflejar la discrepancia entre imaginar que uno tiene un pariente alcohólico y realmente tenerlo. Nuestra muestra de pacientes también ofrece, en términos relativos, una mayor DAP (13\% de los ingresos familiares) que la de la población Suiza.

Las DAP inferiores para los dependientes del alcohol que para sus familiares podría tener diferentes explicaciones. Primero, los ingresos de los pacientes eran menores que los ingresos de los parientes y por lo tanto es de esperar que la DAP fuese inferior. Sin embargo, encontramos que dichas diferencias son muy grandes. Se debe considerar que, mientras que los ingresos personales de los familiares es un $11 \%$ mayor que los de los pacientes, la DAP es más del doble para los dos escenarios. Segundo, las diferencias en la percepción de los problemas generados por la dependencia (los familiares perciben estos problemas como significativamente más severos que los propios dependientes) podrían reflejar otra parte importante de estas diferencias. En otras palabras, estas diferencias podrían estar motivadas en parte 
por diferencias en la ganancia percibida en bienestar. Por último, hay evidencia de que los individuos pueden estar dispuestos a pagar más para evitar un riesgo o tratar la enfermedad de un familiar que para proteger su propia salud (Amin y Khondoker, 2004; Viskusi, Magat y Huber, 1987).

Es discutible si la DAP obtenida está capturando únicamente los costes intangibles, como era nuestro objetivo, o, por el contrario, también está reflejando los costes tangibles (directos o indirectos). Ya que España tiene un sistema de salud pública que requiere un copago mínimo para los servicios, se supone que el coste directo incurrido por el tratamiento de la dependencia no fue incorporado por los entrevistados (o, si es así, sólo marginalmente). Sin embargo, la DAP bien podría estar capturando parte de los costes indirectos resultantes de la pérdida de productividad (pérdida de empleo, menos ingresos por absentismo, pensión de invalidez prematura, etc.). Si bien no sabemos si los participantes consideraron estos efectos cuando dieron su DAP, tenemos información que sugiere que cualquier influencia que pudiera haber tenido era pequeña. Es decir, sólo un $13 \%$ de los pacientes consideraba que el consumo de alcohol había tenido consecuencias graves en su trabajo (si bien este valor aumentó a un 26\% cuando consideramos las opiniones de los familiares).

Hacemos hincapié en la falta de una correlación positiva mayor entre la gravedad de las consecuencias de la dependencia alcohólica y la DAP. Los resultados sugieren que los pacientes con problemas graves dan una DAP significativamente menor que los que tienen problemas moderados. Estos resultados se relacionan con fenómenos muy relevantes para el tratamiento de toxicomanías como, por ejemplo, la percepción de la autoeficacia (Burling, Reilly, Motzen y Ziff, 1989). La autoeficacia tiene que ver con la percepción que el adicto/a tiene de sus posibilidades de éxito y, obviamente, cuanto mayor sean las probabilidades, más estará dispuesto/a a pagar. Es más probable que esto entre en juego para pacientes con problemas menos graves (en las fases muy iniciales, con mayor control de la situación, etc.) y más animados que para pacientes con más problemas que pueden haber fracasado en intentos anteriores de curación o para pacientes que se han adaptado a su situación. La influencia potencial de estos aspectos es evidente en el escenario del $50 \%$ de éxito (los pacientes pueden percibir su probabilidad personal como mayor o menor que la que se ha dado). Sin embargo, los análisis de regresión secundarios indican que estos resultados sólo se mantienen cuando se consideran las respuestas referidas al escenario del $100 \%$ de éxito. Consecuentemente, factores como una menor percepción de la gravedad del problema por pacientes con problemas más graves (y probablemente una adicción más severa) pueden tener una mayor repercusión en estos resultados. Nuestro estudio sugiere que hay mayor disposición a ser tratado entre sujetos alcohólicos en los estadios menos evolucionados de dependencia alcohólica, con apoyo familiar, animados, y cuando no se asocia un gran número problemas secundarios.

Nuestros resultados tienen varias limitaciones. Primero, nuestra muestra de personas con dependencia alcohólica es pequeña y no procede de la población general, lo que podría producir sesgo de selección. Si existe sesgo de selección, no sabemos en qué dirección alteraría la composición de la muestra. Podría haber un sesgo hacia sujetos con dependencia alcohólica más grave, como sería el caso de los que vienen a un centro especializado en el tratamiento de alcoholismo. Sin embargo, el sesgo también podría provenir de la exclusión de pacientes con patología muy severa vinculada en muchos casos a situaciones de exclusión social, que no acuden a tratamiento. En cualquier caso, nuestra muestra tiene algunas ventajas con respecto a una muestra extraída de la población general. Por un lado, nuestro método de reclutamiento garantiza que todos los pacientes entrevistados son dependientes del alcohol, diagnosticados por un especialista. Por otro lado, el tipo de contacto (dentro de una unidad de tratamiento de alcoholismo) y el formato de la entrevista (entrevista directa en vez de un correo electrónico o una entrevista telefónica) proporcionan una tasa de respuesta y un porcentaje de cuestionarios válidos muy elevados en comparación con los que habitualmente se encuentran en este tipo de estudio (Petrie et al., 2008; Saarni et al., 2007), evitando el sesgo que una baja tasa de respuesta podría causar.

Segundo, una porción considerable de los pacientes no tiene familiares comprometidos con el tratamiento. Esto dio lugar a una muestra especialmente pequeña de parientes y podría introducir sesgos de selección que son difíciles de evaluar. Además, el pequeño tamaño de la muestra de familiares puede haber contribuido al resultado que, entre las variables medidas, sólo los ingresos y la probabilidad de éxito del tratamiento influenciaron la DAP. Otra posible limitación es el diseño de las preguntas. Puesto que uno de los escenarios propuso una tasa de curación del 100\%, es posible que los valores de DAP obtenidos estén fuertemente condicionados por las restricciones presupuestarias. Obviamente, cualquier estudio de la DAP se enfrenta a una restricción de presupuesto. Cuando los participantes tienen que indicar cuánto pagarían por un bien, esta cantidad está limitada por sus ingresos y por lo que quieren consumir con los restantes activos. El problema se presenta cuando el beneficio es tan grande que el valor que los participantes asignan al bien supera sus ingresos produciendo una subestimación del beneficio o una insensibilidad de los valores de la DAP a los cambios en la cantidad del bien. Para evitar esto, el alcance del bien objeto de valoración a menudo se reduce con la introducción de, por ejemplo, la probabilidad de obtener el bien de menos del 100\%. En nuestro estudio, se propuso un escenario adicional en el que la probabilidad de éxito era del $50 \%$. El resultado es que los participantes estaban dispuestos a pagar $30 \%$ más para garantizar el éxito 
del tratamiento (37\% más en el caso de familiares). Ya que las diferencias son significativas, creemos que, al menos en la primera pregunta ( $50 \%$ éxito), no se agotó la DAP de los participantes, porque en la segunda pregunta, la cantidad incrementó. La restricción impuesta por la curación del $100 \%$ de éxito es difícil de evaluar. En cualquier caso, nuestros resultados están de acuerdo con la literatura. El estudio de Neuman y Johannesson (1994), por ejemplo, analizando la DAP para un tratamiento de fertilización in vitro, encontró que las participantes estaban dispuestas a pagar entre un $37 \%$ y un $47 \%$ más (dependiendo de la perspectiva) por un programa que garantizaba un $100 \%$ de éxito que por uno que sólo tenía un $50 \%$ de probabilidad de éxito.

Finalmente, la DAP obtenida podría estar influenciada por el formato de pregunta abierta utilizado. Este formato es especialmente conveniente cuando el tamaño de la muestra es pequeño (Carson y Hanemann, 2005), como en nuestro estudio. Sin embargo, hay evidencia empírica de que los tipos de técnicas de elicitación pueden influir en los valores estimados. La literatura relevante indica que los valores obtenidos con un formato abierto o de tarjeta de pago a menudo son inferiores a los resultados de opciones dicotómicas (Gyrd-Hansen, Jensen y Kjaer, 2014). Además, en el área de servicios de salud, se ha encontrado que el formato abierto, comparado con el formato de tarjeta de pago, o produce valoraciones más bajas (Whynes et al., 2003; Donaldson, Thomas y Torgerson, 1997) o no produce diferencias significativas (Gyrd-Hansen et al., 2014). Estos resultados sugieren que nuestro estudio estaría proporcionando valoraciones conservadoras de los costes intangibles de la dependencia alcohólica.

Los resultados obtenidos pueden ser utilizados-con todas las precauciones necesarias dadas las limitaciones mencionadas-en el área de valoración económica, específicamente en estudios de análisis de coste-beneficio. Nuestro estudio proporciona un rango de valores que pueden utilizarse para acercarse a los beneficios derivados de programas centrados en la prevención, tratamiento, o curación de la dependencia alcohólica. Sin embargo, la selección de un solo valor no es fácil, ya que hay que decidir si utilizar los valores de la media o la mediana, los resultados del escenario del $100 \%$ o del $50 \%$ de éxito (en el último escenario, se asume que el beneficio de curar la dependencia es dos veces el valor proporcionado), o, finalmente, las respuestas de los familiares o de los pacientes. Dependiendo de esta decisión, el valor anual para curar un caso de dependencia alcohólica podría oscilar entre $1200 €$-la mediana proporcionada por los pacientes para una tasa de curación del 100\% - hasta 7361€, el doble de la DAP media proporcionada por los familiares para una tasa de curación del $50 \%$. Sugerimos que el beneficio anual de curar (o prevenir) un caso de dependencia alcohólica debería calcularse inicialmente mediante el uso de los valores promedios del escenario del $50 \%$ de éxito (3095€ desde la perspectiva de los pacientes y $7361 €$ desde la perspectiva de los familiares), con un análisis posterior de la sensibilidad utilizando los restantes valores. La razón de esta elección es que el análisis coste-beneficio generalmente utiliza valores promedios y nosotros asumimos que los valores estimados para el escenario de curación del $100 \%$ podrían ser fuertemente restringidos por las restricciones presupuestarias de los participantes. En cualquier caso, estos valores deben tomarse con cautela. Este estudio muestra una metodología para evaluar los costes intangibles y proporciona una primera aproximación a estos valores, pero nuestros hallazgos deben ser validados por estudios futuros con muestras mayores y en otros contextos.

Este estudio sugiere que el enfoque de valoración contingente puede ser un método adecuado para la medición de los costes intangibles derivados de dependencia alcohólica, desde la perspectiva de pacientes y de familiares. Los resultados muestran que las valoraciones obtenidas son muy diferentes, dependiendo de la perspectiva tomada. Si bien una vasta literatura en el área de valoración económica muestra las diferencias entre las perspectivas de los pacientes y la población general, estos resultados añaden nueva evidencia empírica con respecto a las disparidades entre pacientes y familiares. En nuestra opinión, las investigaciones futuras en la medición de los efectos intangibles de la dependencia alcohólica en particular y de drogas en general deben estudiar estas diferencias en mayor profundidad. Ya que los pacientes dependientes pueden distorsionar la verdadera magnitud del problema, la perspectiva de los familiares podría ser especialmente relevante en ese contexto.

\section{Agradecimientos}

Agradecemos la ayuda prestada por los trabajadores de la Unidad de Alcohol de Vigo en el reclutamiento de los pacientes. También agradecemos a todos los pacientes y sus familias que contribuyeron con su tiempo a este estudio. Agradecemos el apoyo financiero del Ministerio Español de Ciencia e Innovación (ECO2015-69334-R) y del Gobierno Regional de Galicia (10SEC300038PR y ECOBAS [AGRUP2015/08]).

\section{Conflicto de intereses}

Los autores declaran que no tienen ningún conflicto de interés en la investigación.

\section{Referencias}

Amin, M. y Khondoker, F. (2004). A contingent valuation study to estimate the parental willingness-to-pay for childhood diarrhea and gender bias among rural households in India. Health Research Policy and Systems, 2, 1359-1386. doi:10.1186/1478-4505-2-3. 
Anderson, P. y Baumberg, B. (2006). Alcohol in Europe. London: Institute of Alcohol Studies. Recuperado de http:// ec.europa.eu/health-eu/news_alcoholineurope_en.htm.

Arrow, K., Solow, R., Portney, P. R., Leamer, E. E., Radner, R. y Schuman, H. (1993). Report of the NOAA Panel on Contingent Valuation. Federal Register 58, 4602-4614.

Baumberg, B. (2010). Best practice in estimating the costs of alcohol-Recommendations for future studies. WHO Regional Office for Europe. Recuperado de http://www.euro.who. int/_data/assets/pdf_file/0009/112896/E93197.pdf .

Bergmo, T. S. y Wangberg, S. C. (2007). Patients' willingness to pay for electronic communication with their general practitioner. The European Journal of Health Economics, 8, 105-110. doi:10.1007/s10198-006-0014-5.

Bishai, D., Sindelar, J., Ricketts, E. P., Huettner, S., Cornelius, L., Lloyd, J. J., ... Strathdee, S. A. (2008). Willingness to pay for drug rehabilitation: implications for cost recovery. Journal of Health Economics, 27, 959-972. doi:10.1016/j.jhealeco.2007.11.007.

Blumenschein, K., Johannesson, M., Yokoyama, K. y Freeman, P. (2001). Hypothetical versus real willingness to pay in the health care sector: results from a field experiment. Value in Health, 4, 79-79. doi:10.1046/j.15244733.2001.40202-36.x.

Boonen, A., Severens, J. L., Van Tubergen, A., Landewé, R., Bonsel, G., Van der Heijde, D. y van der Linden, S. (2005). Willingness of patients with ankylosing spondylitis to pay for inpatient treatment is influenced by the treatment environment and expectations of improvement. Annals of the Rheumatic Diseases, 64, 1650-1652. doi:10.1136/ard.2005.038786.

Brazier, J., Akehurst, R., Brennan, A., Dolan, P., Claxton, K., McCabe, C., ... Tsuchyia, A. (2005). Should patients have a greater role in valuing health states?. Applied Health Economics and Health Policy, 4, 201-208. doi:10.2165/00148365-200504040-00002.

Burling, T. A., Reilly, P. M., Moltzen, J. O. y Ziff, D. C. (1989). Self-efficacy and relapse among inpatient drug and alcohol abusers: a predictor of outcome. Journal of Studies on Alcohol, 50, 354-360. doi:10.15288/jsa.1989.50.354.

Byrne, M. M., O’Malley, K. y Suarez-Almazor, M. E. (2005). Willingness to pay per quality-adjusted life year in a study of knee osteoarthritis. Medical Decision Making, 25, 655-666. doi:10.1177/0272989X05282638.

Carson, R. T. y Hanemann, W. M. (2005). Contingent Valuation. In K. G. Maler \& J. R. Vincent (Eds.), the Handbook of Environmental Economics: Valuing Environmental Changes (Vol. 2), pp 821-920. Elsevier.

Chisholm, D., Rehm, J., Van Ommeren, M. y Monteiro, M. (2004). Reducing the global burden of hazardous alcohol use: a comparative cost-effectiveness analysis. Journal of Studies on Alcohol, 65, 782-793. doi:10.15288/ jsa.2004.65.782.
Collins, D. J. y Lapsley, H. M. (2008). The avoidable costs of alcohol abuse in Australia and the potential benefits of effective policies to reduce the social costs of alcohol (pp. 1-51). Australian Government Department of Health \& Ageing. Recuperado de http://www.nationaldrugstrategy.gov.au/internet/drugstrategy/publishing.nsf/content/mono70.

Corry, J., Sanderson, K., Issakidis, C., Andrews, G. y Lapsley, H. (2004). Evidence-based care for alcohol use disorders is affordable. Journal of Studies on Alcohol. 65, 521-529. doi:10.15288/jsa.2004.65.521.

Diamond, P. A. y Hausman, J. A. (1994). Contingent valuation: Is some number better than no number?. The Journal of Economic Perspectives, 8, 45-64. doi:10.1257/ jep.8.4.45.

Donaldson, C., Thomas, R. y Torgerson, D. J. (1997). Validity of open-ended and payment scale approaches to eliciting willingness to pay. Applied Economics, 29, 79-84. doi:10.1080/000368497327425.

Fautrel, B., Clarke, A. E., Guillemin, F., Adam, V., St-Pierre, Y., Panaritis, T., ... Penrod, J. R. (2007). Costs of rheumatoid arthritis: new estimates from the human capital method and comparison to the willingness-to-pay method. Medical Decision Making, 27, 138-150. doi:10.1016/j. healthpol.2008.12.011.

Fernández, A., Saameño, J. Á. B., Pinto-Meza, A., Luciano, J. V., Autonell, J., Palao, D., ... Serrano, A. (2010). Burden of chronic physical conditions and mental disorders in primary care. The British Journal of Psychiatry, 196, 302309. doi:10.1192/bjp.bp.109.074211.

Gabriel, S. E., Kneeland, T. S., Melton, L. J., Moncur, M. M., Ettinger, B. y Tosteson, A. N. (1999). Health-related Quality of Life in Economic Evaluations for Osteoporosis Whose Values Should We Use?. Medical Decision Making, 19, 141-148. doi:10.1007/s00198-008-0743-7.

García-Pérez, L., Aguiar-Ibáñez, R., Pinilla-Domínguez, P., Arvelo-Martín, A., Linertová, R. y Rivero-Santana, A. (2014). Revisión sistemática de utilidades relacionadas con la salud en España: el caso de la salud mental. Gaceta Sanitaria, 28, 77-83.

Grandes, G., Montoya, I., Arietaleanizbeaskoa, M. S., Arce, V. y Sanchez, A. (2011). The burden of mental disorders in primary care. European Psychiatry, 26, 428-435.

Greenberg, D., Bakhai, A., Neumann, P. J. y Cohen, D. J. (2004). Willingness to pay for avoiding coronary restenosis and repeat revascularization: results from a contingent valuation study. Health Policy, 70, 207-216. doi:10.1016/j.healthpol.2004.03.002.

Gueylard-Chenevier, D. G. y LeLorier, J. (2005). A willingness-to-pay assessment of parents' preference for shorter duration treatment of acute otitis media in children. Pharmacoeconomics, 23, 1243-1255. doi:10.1186/14777525-8-75.

Gyrd-Hansen, D., Jensen, M. L. y Kjaer, T. (2014). Framing the willingness-to-pay question: Impact on response pat- 
terns and mean willingness to pay. Health Economics, 23, 550-563. doi:10.1002/hec.2932.

He, M., Chan, V., Baruwa, E., Gilbert, D., Frick, K. D. y Congdon, N. (2007). Willingness to pay for cataract surgery in rural Southern China. Ophthalmology 114, 411-416. doi:10.1016/j.ophtha.2006.09.012.

Jarl, J., Johansson, P., Eriksson, A., Eriksson, M., Gerdtham, U. G., Hemström, Ö., ... Room, R. (2008). The societal cost of alcohol consumption: an estimation of the economic and human cost including health effects in Sweden, 2002. The European Journal of Health Economics, 9, 351-360. doi:10.1007/S10198-007-0082-1

Jeanrenaud, C. y Pellegrini, S. (2007). Valuing intangible costs of alcohol dependence: A contingent valuation study. Revue d'Economie Politique, 117, 813-825. doi:10.3917/redp.175.0813.

Jimoh, A., Sofola, O., Petu, A. y Okorosobo, T. (2007). Quantifying the economic burden of malaria in Nigeria using the willingness to pay approach. Cost Effectiveness and Resource Allocation, 5, 1. doi:10.1186/1478-7547-5-6.

Johansson, P., Jarl, J., Eriksson, A., Eriksson, M., Gerdtham, U. G., Hemström, Ö., ... Room, R. (2006). The social costs of alcohol in Sweden 2002. Stockholms Universitet, Centrum för socialvetenskaplig alkohol-och drogforskning (SoRAD). doi:10.1007/S10198-007-0082-1.

John, U., Rumpf, H. J., Bischof, G., Hapke, U., Hanke, M. y Meyer, C. (2013). Excess Mortality of Alcohol-Dependent Individuals After 14 Years and Mortality Predictors Based on Treatment Participation and Severity of Alcohol Dependence. Alcoholism: Clinical and Experimental Research, 37, 156-163. doi:10.1111/j.1530-0277.2012.01863.x.

Kraemer, K. L., Roberts, M. S., Horton, N. J., Palfai, T., Samet, J. H., Freedner, N., ... Saitz, R. (2005). Health utility ratings for a spectrum of alcohol-related health states. Medical Care, 43, 541-550. doi:10.1097/01. mlr.0000163644.97251.14.

Laslett, A. M., Catalano, P., Chikritzhs, T., Dale, C., Doran, C., Ferris, J., ... Room, R. (2010). The range and magnitude of alcohol's harm to others. AER Centre for Alcohol Policy Research, Turning Point Alcohol and Drug Centre, Eastern Health: Fitzroy, Victoria.

Maheswaran, H., Petrou, S., Rees, K. y Stranges, S. (2013). Estimating EQ-5D utility values for major health behavioural risk factors in England. Journal of Epidemiology and Community Health, 67, 172-180. doi:10.1136/jech-2012201019.

Mann, R., Brazier, J. y Tsuchiya, A. (2009). A comparison of patient and general population weightings of EQ-5D dimensions. Health Economics, 18, 363-372. doi:10.1002/ hec.1362.

Mortimer, D. y Segal, L. (2005). Economic evaluation of interventions for problem drinking and alcohol dependence: cost per QALY estimates. Alcohol and Alcoholism, 40, 549-555. doi:10.1093/alcalc/agh192
Mosquera J. y Rodríguez-Míguez, E. (2015). Using the SF$6 \mathrm{D}$ to measure the impact of alcohol dependence on health-related quality of life. The European Journal of Health Economics, 16, 347-356. doi:10.1007/s10198-014-0627-z.

Navarro, H. J., Doran, C. M. y Shakeshaft, A. P. (2011). Measuring costs of alcohol harm to others: A review of the literature. Drug and alcohol dependence, 114, 87-99. doi:10.1016/j.drugalcdep.2010.11.009.

Neumann, P. J. y Johannesson, M. (1994). The willingness to pay for in vitro fertilization: a pilot study using contingent valuation. Medical Care, 32, 686-699. doi:10.1186/14726963-8-261

Nutt, D. J., King, L. A. y Phillips, L. D. (2010). Drug harms in the UK: a multicriteria decision analysis. The Lancet, 376, 1558-1565. doi:10.1016/S0140-6736(10)61462-6.

Parrott, S., Godfrey, C., Heather, N., Clark, J. y Ryan, T. (2006). Cost and outcome analysis of two alcohol detoxification services. Alcohol and Alcoholism, 4, 84-91 doi:10.1093/alcalc/agh236.

Petersen, S., Peto, V., Rayner, M., Leal, J., Luengo-Fernández, R. y Gray, A. (2005). European cardiovascular disease statistics: 2005 edition. (pp 70-72). British Heart Foundation. Department of Public Health, University of Oxford.

Petrie, D., Doran, C. y Shakeshaft, A. (2011). Willingness to pay to reduce alcohol-related harm in Australian rural communities. Expert Review of Pharmacoeconomics $\mathcal{E}$ Outcomes Research, 11, 351-363. doi:10.1586/erp.11.28.

Petrie, D., Doran, C., Shakeshaft, A. y Sanson-Fisher, R. (2008). The relationship between alcohol consumption and self-reported health status using the EQ5D: evidence from rural Australia. Social Science E Medicine, 67, 1717 1726. doi:10.1016/j.socscimed.2008.09.017.

Pinto-Prades, J. L., Farreras, V. y de Bobadilla, J. F. (2008). Willingness to pay for a reduction in mortality risk after a myocardial infarction: an application of the contingent valuation method to the case of eplerenone. The European Journal of Health Economics, 9, 69-78. doi:10.1007/s10198007-0041-x.

Rehm, J., Room, R., Monteiro, M., Gmel, G., Graham, K., Rehn, T.,... Jernigan, D. (2004). Comparative quantification of health risks: global and regional burden of disease due to selected major risk factors, World Health Organization. Retrieved at: www.who.int/publications/cra/ chapters/volume1/0959-1108.pdf.

Rehm, J., Rehm, M., Shield, K., Gmel, G. y Gual, T. (2013). Alcohol consumption, alcohol dependence and related harms in Spain, and the effect of treatment-based interventions on alcohol dependence. Adicciones, 25, 11-18. doi:10.1186/1747-597X-8-21.

Saarni, S. I., Suvisaari, J., Sintonen, H., Pirkola, S., Koskinen, S., Aromaa, A. y Lönnqwist, J. (2007). Impact of psychiatric disorders on health-related quality of life: general 
population survey. The British Journal of Psychiatry, 190, 326-332. doi:10.1016/j.comppsych.2008.06.003.

Sadri, H., MacKeigan, L. D., Leiter, L. A. y Einarson, T. R. (2005). Willingness to pay for inhaled insulin. Pharmacoeconomics, 23, 1215-1227. doi:10.1016/j.sapharm.2008. 10.001.

Sanderson, K., Andrews, G., Corry, J. y Lapsley, H. (2004). Using the effect size to model change in preference values from descriptive health status. Quality of Life Research, 13, 1255-1264. doi:10.1023/B:QURE.0000037482.92757.82.

Smith, R. D. (2003). Construction of the contingent valuation market in health care: a critical assessment. Health Economics, 12, 609-628. doi:10.1002/hec.755.

Stouthard, M. E., Essink-Bot, M. L. y Bonsel, G. L. (2000). Disability weights for diseases: a modified protocol and results for a Western European region. The European Journal of Public Health, 10, 24-30. doi:10.1093/eurpub/10.1.24 24-30.

Tang, C. H., Liu, J. T., Chang, C. W. y Chang, W. Y. (2007). Willingness to pay for drug abuse treatment: results from a contingent valuation study in Taiwan. Health Policy, 82, 251-262. doi:10.1016/j.healthpol.2006.09.007.

Ubel, P. A., Loewenstein, G. y Jepson, C. (2003). Whose quality of life? A commentary exploring discrepancies between health state evaluations of patients and the general public. Quality of Life Research, 12, 599-607. doi:10.1023/A:1025119931010.

UKATT Research Team. (2005). Cost effectiveness of treatment for alcohol problems: findings of the randomised UK alcohol treatment trial (UKATT). The British Medical Journal, 331, 544. doi:10.1093/alcalc/agn112.

Unützer, J., Katon, W. J., Russo, J., Simon, G., Von Korff, M., Lin, E., ... Bush, T. (2003). Willingness to pay for depression treatment in primary care. Psychiatric Services, 54, 340-345. doi:10.1176/ps.54.3.340.

Viscusi, W. K., Magat, W. A. y Huber, J. (1987). An investigation of the rationality of consumer valuations of multiple health risks. The RAND Journal of Economics, 18, 465-479.

Walsh, B. M. y Bartfield, J. M. (2006). Survey of parental willingness to pay and willingness to stay for" painless" intravenous catheter placement. Pediatric Emergency Care, 22, 699-703. doi:10.1097/01.pec.0000238743.96606.69.

Whynes, D. K., Frew, E. y Wolstenholme, J. L. (2003). A comparison of two methods for eliciting contingent valuations of colorectal cancer screening. Journal of Health Economics, 22, 555-574. doi:10.1016/S0167-6296(03)00006-7.

Yasunaga, H., Ide, H., Imamura, T. y Ohe, K. (2006). Benefit evaluation of mass screening for prostate cancer: willingness-to-pay measurement using contingent valuation. Urology, 68, 1046-1050. doi:10.1111/j.14422042.2009.02293.x.

Zarkin, G. A., Cates, S. C. y Bala, M. V. (2000). Estimating the willingness to pay for drug abuse treatment: A pilot study. Journal of Substance Abuse Treatment, 18, 149-159. doi:10.1016/S0740-5472(99)00030-6. 